BMJ Surgery, Interventions, $\&$ Health Technologies

\title{
How can robot-assisted surgery provide value for money?
}

\author{
Sejal Patel (D) , ${ }^{1}$ Maroeska M Rovers (D) , ${ }^{1,2}$ Michiel J P Sedelaar, ${ }^{3}$ \\ Petra L M Zusterzeel, ${ }^{4}$ Ad F T M Verhagen, ${ }^{5}$ Camiel Rosman, ${ }^{6}$ \\ Janneke P C Grutters ${ }^{1}$
}

To cite: Patel S, Rovers MM, Sedelaar MJP, et al. How can robot-assisted surgery provide value for money? BMJ Surg Interv Health Technologies 2021;3:e000042. doi:10.1136/ bmjsit-2020-000042

- Additional material is published online only. To view please visit the journal online (http://dx.doi.org/10.1136/ bmjsit-2020-000042).

Received 06 April 2020 Revised 22 November 2020 Accepted 14 December 2020

Check for updates

(c) Author(s) (or their employer(s)) 2021. Re-use permitted under CC BY-NC. No commercial re-use. See rights and permissions. Published by BMJ.

${ }^{1}$ Department of Operating Rooms, Radboudumc, Nijmegen, Gelderland, The Netherlands

${ }^{2}$ Operating Rooms and Health Evidence, Radboud Universiteit, Nijmegen, The Netherlands

${ }^{3}$ Department of Urology,

Radboudumc, Nijmegen, Gelderland, The Netherlands

${ }^{4}$ Department of Obstetrics and Gynecology, Radboudumc, Nijmegen, Gelderland, The

Netherlands

${ }^{5}$ Department of Cardiothoracic Surgery, Radboudumc,

Nijmegen, Gelderland, The Netherlands

${ }^{6}$ Department of Surgery, Radboudumc, Nijmegen, Gelderland, The Netherlands

Correspondence to

Sejal Patel;

sejal.patel@radboudumc.nl

\section{ABSTRACT}

Objectives To develop an interactive tool that estimates what potential benefits are needed for the robot to provide value for money when compared with endoscopic or open surgical interventions.

Design A generic online interactive tool was developed to analyze the (health) effects needed to compensate for the additional costs of using a surgical robotic system from a healthcare perspective. The application of the tool is illustrated with a hypothetical new surgical robotic platform. A synthesis of evidence from different sources was used combined with interviews with surgeons. Setting Flexible tool that can be adapted to flexible settings.

Participants Any hospital patient group for which robotic, endoscopic or open surgical procedures may be considered as appropriate treatment alternatives (eg, urology, gynecology, and so on).

Intervention Robotically assisted surgical interventions. Comparator Endoscopic or open surgical interventions. Main outcome measures Thresholds of how much (health) effect is needed for robot-assisted surgery to provide value for money and to become cost-effective. Results The utilization rate of the surgical robotic system and a reduction in complications appeared to be important aspects in determining the value for money. To become cost-effective, it was deemed important for new surgical robotic systems to have added clinical benefit and become less costly than the current system.

Conclusions This paper and its assisting interactive tool can be used by clinicians, researchers, and policymakers to gain insight in the benefit needed to provide value for money when using a (new) surgical robotic system or, when the effects are known or can be estimated, to assess the value for money for a specific indication. For robotic surgery to provide most value for money, we recommend assessing for each indication whether the necessary effects seem achievable.

\section{INTRODUCTION}

Over the past decades, the implementation of robot-assisted surgery has increased tremendously. In 2000, about 1000 robot-assisted procedures were performed worldwide, whereas in 2018, that number had increased to more than 1 million. ${ }^{1}$ Besides an increase in the number of robot-assisted procedures performed, an expansion in types of

\section{Short summary}

What is already known about this subject?

- Over the past decades, the number of robot-assisted procedures has increased tremendously, despite the high cost. Combined with the rising pressure on the sustainability of healthcare, this has led to a vicarious debate about its value for money.

\section{What are the new findings?}

- As different surgical robotic systems may have many different features and can be used for multiple indications, we developed an online tool that can be used to analyze the (health) effects needed to compensate for the additional costs of using a surgical robotic system, in order to become cost-effective. The model provides relevant stakeholders, such as surgeons, researchers, purchasers, and policymakers, the unique opportunity to gain insight in what is needed to provide value for money when using (new) surgical robotic systems.

\section{How might these results affect future} research or surgical practice?

- Our model offers guidance for future research as it can be estimated which indications have the potential to become cost-effective and therefore should be further investigated.

procedures is seen. The first robotic systems were mainly used in urology (prostatectomy) and gynecology (hysterectomy). Nowadays, we see a huge increase in application in other fields such as general, gastrointestinal, and thoracic surgery. ${ }^{2}$ With the ever increasing pressure on sustainable healthcare, it is important to know how a surgical robotic system can be used most cost-effectively.

Currently, the da Vinci Surgical System of Intuitive is with 5114 installed systems worldwide the best known and most used system. ${ }^{3}$ Ever since the introduction of da Vinci Surgical System there has been both enthusiasm and skepticism, followed by a vicarious debate on its value for money. Reported effects of robot-assisted surgery include less conversions, reduced blood loss, fewer 
perioperative complications, shorter hospital stays, faster recovery, and less positive tumor resection margins, ${ }^{4-6}$ but these advantages are not confirmed in all studies and for all procedures. Moreover, these advantages come at a high financial investment. ${ }^{1}$ Hospitals spend between $\$ 1000$ and $\$ 4000$ more per robot-assisted case compared with endoscopic minimally invasive or open procedures, in addition to the purchase and maintenance costs of the surgical robotic system. ${ }^{7}$ Barbash and Glied estimated that if robot-assisted procedures replaced all conventional procedures, an additional $\$ 2.5$ billion would be spent in annual healthcare costs in the USA. ${ }^{7}$

Sustainability of healthcare is an important issue in all countries. The increasing number of innovation entering the market demands healthcare providers to prioritize their investments in order to manage increasing costs and deliver the best possible outcomes for patients. Therefore, identifying which innovations provide most value for money is becoming increasingly important. As evidence regarding the cost-effectiveness of robot-assisted surgery is still lacking, the use of robotic surgery is becoming an important societal issue. In order to provide value for money, the increased costs of robot-assisted surgery need to be outweighed by the benefits. It is expected that in the coming years more providers (eg, Stryker, Johnson \& Johnson, Medtronic and smaller companies) will increase their market share or enter the market with new surgical platforms leading to a further increase in robotassisted surgeries in daily practice. With more providers of surgical robotic systems on the market, competing with Intuitive for new customers, it is expected that less expensive systems will become available and that robot-assisted surgery will be applied for a wider range of procedures. ${ }^{7}$ However, the question remains whether such new systems will provide value for money.

As different surgical robotic systems may have many different features and can be used for multiple indications, we aimed to develop a generic online interactive tool that can be used to analyze the (health) effects needed to compensate for the additional costs of using a surgical robotic system, in order to become cost-effective. This tool can be used to explore under which circumstances a (new) surgical robotic system could provide value for money and to inform clinical research. In this paper, we will describe the tool and illustrate its application using a hypothetical new surgical robotic system. The tool enables adjustment of all input variables (ie, acquisition costs robotic system, number of procedures yearly performed) for different surgical robotic systems, and different hospital settings and indications.

\section{MODEL CONSTRUCTION}

\section{Model structure}

A health economic model was developed to estimate the thresholds of how much (health) effect is needed to outweigh the additional costs of a robot-assisted procedure compared with endoscopic and open procedures in a generic patient population over the life course of an individual. The costs and potential effects of these procedures are included in the model. In the model, robotic surgery can be compared with endoscopic or an open procedure, depending on the users' procedure of interest. The analysis was conducted from a healthcare perspective, including all relevant medical costs incurred for the health system. The model incorporates six aspects on which a robot-assisted procedure can potentially provide (health) effect for patients compared with an endoscopic or open procedure. These aspects were based on scientific literature and interviews with experts $(n=4)$ operating in the field of urology, gynecology, thoracic and gastrointestinal surgery. These aspects are procedure time, conversion rate, complications, positive resection margins, length of stay and quality of life. The total costs, consisting of the fixed and variable costs, of a robotassisted procedure and an endoscopic or open procedure can be calculated in the model. Subsequently, the additional costs of a procedure with a surgical robotic system are compared with the possible benefits that can be gained with a robot-assisted procedure. Besides possible benefits, also negative consequences of performing a robot-assisted procedure (eg, longer duration of procedure) can be entered into the model. The model allows for adjustments for specific procedures and specific populations, therefore one can use the model to calculate the benefits needed for a specific procedure and population. Where possible, we followed the Consolidated Health Economic Evaluation Reporting Standards (CHEERS) guidelines. ${ }^{4}$

The interactive model comprises an open web-based tool created with the Shiny package for $\mathrm{R}^{8}$ The online tool allows users to assess the extra costs and benefits of a surgical robotic system for a specific procedure of interest by entering data and/or costs for this specific procedure. A user manual is available to make sure that the tool can also be used without the background information in this article.

\section{Costs procedure}

The costs of a procedure are divided in fixed and variable costs. The fixed costs were defined as the acquisition costs for surgical devices, that is, a surgical robotic system or an endoscopic tower. The annual capital costs of the surgical robotic system and endoscopy tower were calculated by dividing the initial costs for equipment acquisition by an annuity factor, taking into account the useful life years of the equipment and the interest rate. ${ }^{5}$ To calculate the total annual capital costs, the yearly costs for maintaining the equipment were added. To calculate the costs per procedure the total annual capital costs were divided by the number of yearly performed procedures with a surgical robotic system or endoscopy. The variable costs consist of the additional costs for disposables. The equations for cost calculation can be found in the online supplemental appendix II. Costs are presented in euros. 


\section{Potential benefits}

In order to compare the potential effects of a robotassisted procedure with the additional costs of the procedure, each aspect in the model is associated with a monetary value. Procedure time was defined as the operating room costs per minute based on cost calculations of a university medical center (Radboudumc), including three operating room assistants, an anesthesiologist and a surgeon. Despite the widespread adoption of endoscopic and robotic surgeries, there are still patients who require conversion to laparotomy during surgery. When compared with endoscopic surgery, a robot-assisted procedure may result in a lower percentage of conversions. The costs of a conversion are procedure specific and can be entered into the model for a procedure of interest. A robot-assisted procedure could also result in fewer complications, which are in the model divided into short-term complications (ie, within 30 days) and long-term complications (ie, after 30 days). The short-term complications, classified according to the Clavien-Dindo classification system, are subdivided into mild (Clavien-Dindo grade I/ II) and severe (Clavien-Dindo grade III-V) ${ }^{9}$ Long-term complications are defined as lasting complications for which prolonged treatment is required. As the costs for complications may vary widely for different indications these can be adapted in the model for the procedure of interest. Furthermore, performing a robot-assisted procedure may result in fewer positive tumor resection margins when compared with an endoscopic or open procedure. The consequences and, therefore, costs of a positive tumor resection margin are indication specific, which can also be entered into the interactive model for the procedure of interest. When compared with open surgery, patients undergoing robot-assisted surgery may be hospitalized for a shorter period. The reference costs for hospital stay per day were obtained from the Dutch costing manual. ${ }^{5}$ Quality of life was operationalized by means of quality-adjusted life years (QALY), which are a measure combining quality of life and survival, where a QALY of 1 represents living 1 year in perfect health. ${ }^{6}$ The cost-effectiveness threshold was set on $€ 50.000$ per QALY, ${ }^{9}$ but can be changed in the model.

\section{Analysis}

The additional costs of a robot-assisted procedure were calculated by subtracting the total costs of the chosen comparator, an endoscopic or open procedure, from the total costs of the robot-assisted procedure. Threshold analysis for six aspects incorporated in the model is performed. This means that for every aspect the minimum effect that is needed to be achieved by performing a robot-assisted procedure to outweigh the additional costs is calculated, that is, the number of minutes of operating time or hospital days that need to be prevented to outweigh the extra costs; the rate of conversions, complications or positive margins that need to be prevented to outweigh the extra costs; the number of QALYs that need to be gained to become cost-effective. These thresholds were calculated by dividing the additional costs of a robotic procedure with the monetary value of each aspect.

We provided a hypothetical example for a procedure performed with a surgical robotic system compared with laparoscopy to illustrate how the model can be used. For this hypothetical example, we will calculate the required effects for a robot-assisted procedure to compensate the additional costs. Furthermore, to illustrate the use of the tool we showed three possible scenarios in which the surgical robotic system becomes cost-effective. Additionally, we assessed the impact of the acquisition price of the surgical robotic system and the number of procedures performed per year.

\section{MODEL OUTPUT}

The model can be used in two ways. First, the model shows the additional costs of performing a robot-assisted procedure and the effect needed on each aspect to compensate for these additional costs, for example, the number of hospital days that need to be prevented per procedure to offset the extra costs of a procedure with a surgical robotic system. Second, when the effects of a robot-assisted procedure are known, for example, a reduction in procedure time, they can be entered into the model. The model will show if the additional costs for a robotic procedure are compensated by the benefits. In case the (expected) benefits do not (yet) compensate for the additional costs of a robot-assisted procedure, it shows how much extra effect on each aspect is needed to outweigh the additional costs.

\section{CLINICAL EXAMPLE}

To illustrate how the model can be used, we will describe a hypothetical example for a procedure performed with a surgical robotic system compared with laparoscopy from a healthcare perspective. We would like to emphasize that the examples we used are all hypothetical to explain how the tool can be used, and do not represent real examples from clinical practice. For the costs of the robot-assisted procedure we assumed a new surgical robotic system will be used with an acquisition price of $€ 1$ million and a maintenance charge of $€ 80000$ per year. For the endoscopic procedure, acquisition cost for the endoscopy tower of $€ 100000$ and a maintenance charge of $€ 8000$ per year were assumed. For both devices, we assumed a useful life of 7 years and an interest rate of $4.2 \% .^{5}$ An average of 200 procedures per year for both robot-assisted and endoscopic surgeries were used to calculate the fixed costs per procedure (table 1). We assumed variable costs of $€ 1500$ and $€ 1000$ for the robot-assisted and endoscopic procedures, respectively (table 1 ). The assumed costs of a conversion, complications and a positive surgical margin are presented in table 1 . Since we used a hypothetical example to explain the model, the population is not relevant. When one uses the model to a specific procedure the population needs to be specified. 
Table 1 Input costs for a hypothetical example of a procedure with a new surgical robotic system

\begin{tabular}{|c|c|c|}
\hline Variable & Value & Source \\
\hline \multicolumn{3}{|l|}{ Robot-assisted procedure } \\
\hline \multicolumn{3}{|l|}{ Fixed cost } \\
\hline New surgical robotic system & $€ 1000000$ & Assumption* \\
\hline Useful life surgical robotic system (in years) & 7 & Assumption* \\
\hline Annual interest rate & $4.20 \%$ & 5 \\
\hline Annual maintenance cost & $€ 80000$ & Assumption* \\
\hline Annual number of procedures & 200 & Assumption* \\
\hline Fixed cost per procedure & $€ 1239$ & \\
\hline \multicolumn{3}{|l|}{ Variable cost } \\
\hline Variable cost robotic system & $€ 1500$ & Assumption* \\
\hline Total cost & $€ 2739$ & \\
\hline \multicolumn{3}{|l|}{ Endoscopic procedure } \\
\hline \multicolumn{3}{|l|}{ Fixed cost } \\
\hline Endoscopy tower & $€ 100000$ & Radboudumc \\
\hline Useful life endoscopy tower (in years) & 7 & Radboudumc \\
\hline Annual interest rate & $4.20 \%$ & 5 \\
\hline Annual maintenance cost & $€ 8000$ & Assumption* \\
\hline Annual number of procedures & 200 & 5 \\
\hline Fixed cost per procedure & $€ 124$ & \\
\hline \multicolumn{3}{|l|}{ Variable cost } \\
\hline Variable cost endoscopic procedure & $€ 1000$ & Assumption* \\
\hline Total cost & $€ 1124$ & \\
\hline \multicolumn{3}{|l|}{ Benefit for potential effects } \\
\hline Duration of operation (min) & $€ 13.93$ & Radboudumc \\
\hline Conversion & $€ 1700$ & $18-20^{*}$ \\
\hline Complication with Clavien-Dindo I/II & $€ 5000$ & $18-20^{*}$ \\
\hline Complication with Clavien-Dindo III-V & $€ 16000$ & $18-20^{\star}$ \\
\hline Lasting complication & $€ 15000$ & $18-20^{*}$ \\
\hline Positive margin & $€ 5000$ & $18-20^{*}$ \\
\hline Hospitalization (day) & $€ 668$ & 5 \\
\hline QALY & $€ 50000$ & 9 \\
\hline
\end{tabular}

*We illustrated the use of the model with a hypothetical example, for which we used the input values given in this table. Even though these input values were based on existing literature, these do not reflect a specific indication.

QALY, quality-adjusted life year.

For this example, the total extra costs for a robotassisted procedure compared with laparoscopy were $€ 1615$ (€2739 minus $€ 1124$ ). To compensate for these extra costs, robot-assisted surgery should result in either a shorter operating time of $116 \mathrm{~min}$, or a reduction in conversions of $95 \%$, or a reduction of $32 \%$ of ClavienDindo I/II complications, or prevent $10 \%$ of ClavienDindo III-V complications, or prevent $32 \%$ of lasting complications, or reduce positive margins with $32 \%$, or a reduction of 2.42 hospital days, or gain 0.032 QALYs (table 2). This means that, for example, 0.032 QALYs should be gained with robotic surgery compared with laparoscopic surgery to compensate for the extra costs of $€ 1615$ per robot-assisted procedure (table 2), when assuming a willingness to pay of $€ 50000$ per QALY (table 1).

A surgical robotic system may provide effect on more than one aspect at a time. Therefore, a combination of effects seen or expected for a procedure can be entered into the model to determine if these would compensate for the extra costs involved with a robot-assisted procedure or if extra benefit is still needed. Table 3 provides three scenarios for which the effects would outweigh the extra costs of the procedure when those effects can be 
Table 2 Required effects for a robot-assisted procedure to compensate the additional costs if these effects would occur in only one variable at a time

Effect needed to
compensate the additional
costs of $€ 1615$ per robot-
assisted procedure
compared with laparoscopy*
-116
$-95 \%$
$-32 \%$
$-10 \%$
$-11 \%$
$-32 \%$
-2.24
0.032

*The percentages in the table are absolute percentages, the percentage of patients in the total group of patients in which this must be prevented.

QALY, quality-adjusted life year.

achieved using the surgical robotic system. When a reduction of 25 min operating time, as well as a reduction of $10 \%$ for complications with Clavien-Dindo I/II, and $5 \%$ for a Clavien-Dindo III-V can be achieved using the surgical robotic system, the benefits of these effects will outweigh the extra costs of the procedure (scenario I). A reduction of one hospital day together with a QALY gain of 0.02 will also result in sufficient benefit to outweigh the extra costs of a robot-assisted procedure (scenario II). Scenario III shows an example in which a combination of effect on operating time, conversion, complications and positive margin could result in compensating the additional costs of a robotic procedure. Scenario IV provides an example in which besides positive consequences, also
Table 4 The additional cost per procedure with the surgical robotic system when either the acquisition costs for the surgical robotic system or the number of procedures per year is varied

Extra cost per procedure when comparing the robot with laparoscopy

Acquisition of surgical robotic $€ 1086$
system of $€ 500000$
$\begin{aligned} & \text { Acquisition of surgical robotic } \\ & \text { system of } € 2500000\end{aligned} 52764$
$\begin{aligned} & 100 \text { procedures per year } \\ & \text { performed }\end{aligned}$
$\begin{aligned} & 300 \text { procedures per year } \\ & \text { performed }\end{aligned}$

a negative consequence, a longer operating time of 15 min, for a robotic procedure is included.

The additional costs for a robot-assisted procedure are dependent on various inputs such as the acquisition costs of the surgical robotic system and the number of procedures performed per year (online supplemental appendix I figures 1 and 2). The model can be used to assess the impact of such changes on the value for money. Table 4 shows the influence of the additional costs of a robot-assisted procedure when the acquisition costs of the surgical robotic system were varied from $€ 500000$ to $€ 2$ 500000 and the number of procedures was varied from 100 to 300 . In case the costs of the surgical robotic system are reduced to $€ 500000$, the additional costs per procedure for our example were $€ 1086$ as compared with laparoscopy. The effects needed for each individual aspect to compensate for these extra costs are presented in table 5 . The results show that a $7 \%$ reduction in complications with Clavien-Dindo III-V is sufficient to compensate for these additional costs. On the other hand, when performing 100 robot-assisted procedures per year the additional costs per procedure resulted in $€ 4598$ as compared with

Table 3 Hypothetical examples of combinations of effects that would outweigh the additional costs of a robot-assisted procedure when compared with laparoscopy

\begin{tabular}{lcccc}
\hline & Scenario I* & Scenario II & Scenario III* & Scenario IV $^{*}$ \\
\hline Duration of operation (min) & -25 & 0 & -5 & +15 \\
Conversion & 0 & 0 & $-2 \%$ & 0 \\
Complication with CD I/II & $-10 \%$ & 0 & $-5 \%$ & $-5 \%$ \\
Complication with CD III-V & $-5 \%$ & 0 & $-5 \%$ & $-3 \%$ \\
Lasting complication & 0 & 0 & $-5 \%$ & $-3 \%$ \\
Positive margin & 0 & 0 & $-3 \%$ & 0 \\
Hospitalization (day) & 0 & -1 & 0 & -1 \\
QALY & 0 & 0.02 & 0 & 0 \\
\hline
\end{tabular}

*The percentages in the table are absolute percentages, the percentage of patients in the total group of patients in which this must be prevented.

CD, Clavien-Dindo; QALY, quality-adjusted life year. 
Table 5 Required effects with the robot to compensate the minimum and maximum additional costs (table 4) if these effects would occur in only one variable at a time

\begin{tabular}{|c|c|c|}
\hline & $\begin{array}{l}\text { Effect needed } \\
\text { per variable to } \\
\text { compensate the } \\
\text { additional costs of } \\
€ 1086^{\star}\end{array}$ & $\begin{array}{l}\text { Effect needed } \\
\text { per variable to } \\
\text { compensate } \\
\text { the additional } \\
\text { costs of } \\
€ 4598^{\star}\end{array}$ \\
\hline $\begin{array}{l}\text { Duration of operation } \\
\text { (min) }\end{array}$ & -79 & -335 \\
\hline Conversion & $-64 \%$ & N/A (>100\%) \\
\hline $\begin{array}{l}\text { Complication with } \\
\text { Clavien-Dindo I/II }\end{array}$ & $-22 \%$ & $-92 \%$ \\
\hline $\begin{array}{l}\text { Complication with } \\
\text { Clavien-Dindo III-V }\end{array}$ & $-7 \%$ & $-29 \%$ \\
\hline Lasting complication & $-7 \%$ & $-31 \%$ \\
\hline Positive margin & $-22 \%$ & $-92 \%$ \\
\hline Hospitalization (day) & -1.63 & -6.88 \\
\hline QALY & 0.022 & 0.092 \\
\hline
\end{tabular}

*The percentages in the table are absolute percentages, the percentage of patients in the total group of patients in which this must be prevented. N/A (>100\%) means that more than $100 \%$ prevention is warranted to compensate for the extra costs of a robot-assisted procedure.

QALY, quality-adjusted life year.

laparoscopy. A reduction of $29 \%$ in complications with Clavien-Dindo III-V is then needed to compensate the additional costs of the procedure.

\section{DISCUSSION}

As far as we are aware, we are the first offering a generic and flexible insight in the potential cost-effectiveness of (new) surgical robotic systems. This is particularly important in the current era where many new systems are being developed while the affordability of healthcare is increasingly under pressure. The interactive model described in this paper provides relevant stakeholders, such as surgeons, purchasers, and policymakers, the unique opportunity to gain insight in what is needed to provide value for money when using (new) surgical robotic systems. It informs them on the effect that is needed to compensate the additional costs of a (new) surgical robotic system.

Earlier studies showed that surgical costs and effects for robot-assisted procedures may vary between indications. ${ }^{10}{ }^{11}$ Gkegkes $e t a l$, for example, found ranges from $€ 2539$ to $€ 57002$ and from $€ 7888$ to $€ 16851$ for robotassisted and endoscopic procedures, respectively. ${ }^{10}$ The costs used in our analysis, €2739 for a robot-assisted procedure and $€ 1124$ for an endoscopic procedure, seem low compared with these costs. This can be explained by the fact that the current surgical robotic system is more expensive than the costs we assumed for the new hypothetical surgical robotic system in the present study. Furthermore, we only included the fixed and variable costs of the equipment used and did not include other costs such as operating theater costs and anesthesia.

Some potential limitations of our model should also be discussed. First, less blood loss is often mentioned as a benefit for robot-assisted procedures, which we did not take into account in our model as we only focused on patient-related outcomes. However, blood loss may impact complications, which were incorporated in the model. Second, we also did not include potential ergonomic benefits for the surgeon in the model. Studies have shown that the ergonomics for the surgeon associated with robotic surgery is better compared with laparoscopic surgery. ${ }^{12-14}$ Ergonomic problems may affect a surgeon's physical workload and potentially may even lead to absenteeism. As the costs of absenteeism of a surgeon are estimated at $€ 1129$ per day, ${ }^{5}$ while the extra costs per procedure with the robot are $€ 2345$, a saving of more than 2 days of absenteeism per procedure performed is needed to compensate the extra costs. However, although it is very relevant, this issue is difficult to quantify. Quality of life of the surgeon can be one of the reasons, next to value for money, for using or purchasing a surgical robotic system. Third, we did not include learning curve effects and a potential increase in procedures that can be performed with future robotic systems that cannot be performed endoscopically yet. Especially for procedures that are performed with open surgery, robotic systems might affect the costs as well as the volume of procedures, since more patients could be treated on 1 hour. Furthermore, due to advances in technology, it is expected that in the future the duration of learning curves will reduce for robot-assisted surgery. ${ }^{15}$ Furthermore, we only focused on including costs from a healthcare perspective. Productivity gains for patients who can return to work more rapidly were not taken into account. Also, we did not look at the reimbursement hospitals will receive from insurers for the additional hospital spending for robotic surgery. Therefore, we cannot provide insight in the value for money of robotic surgery from a societal or hospital perspective.

We aimed to follow the international CHEERS reporting guideline. However, as we did not conduct a standard cost-effectiveness study, some aspects were not applicable for our model. One should be aware that when using our model to perform a cost-effectiveness analysis, it is advised to follow all components of the guideline.

The clinical implication of our model is that more studies into the effectiveness of new surgical robotic systems seem warranted as these systems might become cost-effective, but only if they indeed also have added clinical benefits and if future systems become less costly. The many hospitals that already adopted a surgical robotic system can use the model to explore how the system should be used to provide most value for money by changing the costs and effects to their specific setting. As there is a lot of variation between indications, our model can also easily be used to assess if the expected effects for an indication will provide 
sufficient benefit to provide value for money. In this way, the model can be used to prioritize research.

Hospitals may also have other reasons for investing in costly new advanced technology, for example, scientific purposes, patient demand, fear of missing out, and competition between hospitals. ${ }^{16}{ }^{17}$ On the other hand, we believe that in the current era of rising healthcare costs, hospitals and other relevant stakeholders have to educate patients and society that new is not always better, and that money should be spent wisely. Our interactive model helps them in this discussion.

The utilization rate of a surgical robotic system showed a large influence on the extra cost per procedure. Increasing the number of procedures in our example, from 100 to 300 per year, appeared to reduce the extra costs per procedure with $€ 3005$ when compared with laparoscopy. This might lead to the clinical implication that the number of procedures with the robotic system should be as high as possible. However, the additional variable costs per robot-assisted procedure have to be compensated by a minimum health gain, otherwise the total costs per year will increase even though the fixed costs per procedure will decrease when more procedures are performed. For this reason, it is important to study specific indications and to assess whether the variable costs are indeed compensated by a health gain for that specific indication. Setting the fixed costs in the tool to zero can help explore whether this is the case.

In conclusion, our model and interactive tool provide a unique opportunity for all stakeholders such as researchers, clinicians and policymakers, to gain insight in the benefit needed to outweigh the additional costs of a (new) surgical robotic system or, when the benefits are known or can be estimated, to assess the value for money for a specific procedure. To achieve the maximum value for money, we recommend assessing for each indication whether the necessary effects seem feasible. The online tool (see also: https://sejal.shinyapps.io/supplement_ robot-assisted_surgery_article/) provides this information, and can be easily used by all stakeholders.

\section{Twitter Maroeska M Rovers @MaroeskaRovers}

Contributors Conception or design of the work: SP, MMR, MJPS, PLMZ, AFTMV, CR, JPCG. Data collection: SP. Data analysis and interpretation: SP, MMR, JPCG. Drafting the article: SP. Critical revision of the article: MMR, MJPS, PLMZ, AFTMV, CR, JPCG. Final approval of the version to be published: MMR, MJPS, PLMZ, AFTMV, CR, JPCG.

Funding The authors have not declared a specific grant for this research from any funding agency in the public, commercial or not-for-profit sectors.

Competing interests MMR, JPCG and SP have received an unrestricted research grant from Siemens Healthineers for other but related research projects.

\section{Patient consent for publication Not required.}

Ethics approval Ethics approval and informed consent were not required for this study, since we solely gathered expert opinion on the model structure from the collaborating clinicians.

Provenance and peer review Not commissioned; externally peer reviewed.

Data availability statement All data relevant to the study are included in the article or uploaded as supplementary information. Link to online tool: https://sejal. shinyapps.io/supplement_robot-assisted_surgery_article/.
Open access This is an open access article distributed in accordance with the Creative Commons Attribution Non Commercial (CC BY-NC 4.0) license, which permits others to distribute, remix, adapt, build upon this work non-commercially, and license their derivative works on different terms, provided the original work is properly cited, appropriate credit is given, any changes made indicated, and the use is non-commercial. See: http://creativecommons.org/licenses/by-nc/4.0/.

\section{ORCID iDs}

Sejal Patel http://orcid.org/0000-0002-5858-5166

Maroeska M Rovers http://orcid.org/0000-0002-3095-170X

\section{REFERENCES}

1 Intuitive Surgical. Q2 Investor presentation, 2019. Available: https://isrg.gcs-web.com/static-files/0d3d24c1-849d-4495-8035c17def42fd76 [Accessed 04 Jul 2018].

$2 \mathrm{Ng} \mathrm{ATL}$, Tam PC. Current status of robot-assisted surgery. Hong Kong Med J 2014;20:241-50.

3 Intuitive Surgical. Intuitive Announces first quarter earnings. intuitive surgical, 2019. Available: https://isrg.gcs-web.com/news-releases/ news-release-details/intuitive-announces-first-quarter-earnings [Accessed 15 May 2019].

4 Husereau D, Drummond M, Petrou S, et al. Consolidated Health Economic Evaluation Reporting Standards (CHEERS)--explanation and elaboration: a report of the ISPOR Health Economic Evaluation Publication Guidelines Good Reporting Practices Task Force. Value Health 2013;16:231-50.

5 Zorginstituut Nederland. Costing manual: methodology of research costs and reference prices for economic evaluations in health care, 2016. Available: https://www.zorginstituutnederland.nl/publicaties/ publicatie/2016/02/29/richtlijn-voor-het-uitvoeren-van-economischeevaluaties-in-de-gezondheidszorg [Accessed 25 Jul 2018].

6 Neumann PJ, Cohen JT. QALYs in 2018-Advantages and concerns. JAMA 2018;319:2473-4.

7 Barbash GI, Glied SA. New technology and health care costs the case of robot-assisted surgery. $N$ Engl J Med Overseas Ed 2010;363:701-4.

8 R Core Team. R: a language and environment for statistical computing. Vienna, Austria: R Foundation for Statistical Computing, 2017. https://www.R-project.org/. (accessed August 8, 2019).

9 Versteegh MM, Ramos IC, Buyukkaramikli NC, et al. SeverityAdjusted probability of being cost effective. Pharmacoeconomics 2019;37:1155-63.

10 Gkegkes ID, Mamais IA, lavazzo C. Robotics in general surgery: a systematic cost assessment. J Minim Access Surg 2017;13:243-55.

11 Bolenz C, Gupta A, Hotze T, et al. Cost comparison of robotic, laparoscopic, and open radical prostatectomy for prostate cancer. Eur Urol 2010;57:453-8.

12 van der Schatte Olivier RH, Van't Hullenaar CDP, Ruurda JP, et al. Ergonomics, user comfort, and performance in standard and robotassisted laparoscopic surgery. Surg Endosc 2009;23:1365-71.

13 Lawson EH, Curet MJ, Sanchez BR, et al. Postural Ergonomics during robotic and laparoscopic gastric bypass surgery: a pilot project. J Robot Surg 2007;1:61-7.

14 Van't Hullenaar CDP, Bos P, Broeders IAMJ. Ergonomic assessment of the first assistant during robot-assisted surgery. J Robot Surg 2019;13:283-8.

15 Royal College of Surgeons England. Future of surgery, 2019. Available: https://futureofsurgery.rcseng.ac.uk/ [Accessed $20 \mathrm{Sep}$ 2019].

16 Aggarwal A, Lewis D, Mason M, et al. Effect of patient choice and hospital competition on service configuration and technology adoption within cancer surgery: a national, population-based study. Lancet Oncol 2017;18:1445-53.

17 Abrishami P, Boer A, Horstman K. When the evidence basis breeds controversies: exploring the value profile of robotic surgery beyond the early introduction phase. medical care research and review. MCRR, 2019: 1077558719832797.

18 Gomez-Rosado J-C, Salas-Turrens J, Olry-de-Labry-Lima A. Economic cost analysis related to complications in general and digestive surgery. Cir Esp 2018;96:292-9.

19 Michels CTJ, Wijburg CJ, Leijte E, et al. A cost-effectiveness modeling study of robot-assisted (RARC) versus open radical cystectomy (ORC) for bladder cancer to inform future research. Eur Urol Focus 2019;5:1058-65.

20 Widmar M, Strombom P, Keskin M, et al. Burden of surgical complications: contribution of long-term costs by Clavien-Dindo classification. J Am Coll Surg 2016;223:e90. 